\title{
Probabilistic eigensolver with a trapped-ion quantum processor
}

\author{
Jing-Ning Zhang $\odot,{ }^{1,2, *}$ Iñigo Arrazola $\odot,{ }^{3}$ Jorge Casanova $\odot,{ }^{3,4}$ Lucas Lamata $\odot,{ }^{3,5}$ Kihwan Kim $\odot,{ }^{2}$ \\ and Enrique Solano $\oplus^{3,4,6,7, \dagger}$ \\ ${ }^{1}$ Beijing Academy of Quantum Information Sciences, Beijing 100193, China \\ ${ }^{2}$ Center for Quantum Information, Institute for Interdisciplinary Information Sciences, Tsinghua University, \\ Beijing 100084, People's Republic of China \\ ${ }^{3}$ Department of Physical Chemistry, University of the Basque Country UPV/EHU, Apdo. 644, 48080 Bilbao, Spain \\ ${ }^{4}$ IKERBASQUE, Basque Foundation for Science, Maria Diaz de Haro 3, 48013 Bilbao, Spain \\ ${ }^{5}$ Departamento de Física Atómica, Molecular y Nuclear, Universidad de Sevilla, 41080 Sevilla, Spain \\ ${ }^{6}$ International Center of Quantum Artificial Intelligence for Science and Technology (QuArtist) \\ and Department of Physics, Shanghai University, 200444 Shanghai, China \\ ${ }^{7}$ IQM, Munich, Germany
}

(Received 4 April 2020; accepted 7 April 2020; published 18 May 2020)

\begin{abstract}
Preparing the eigenstate, especially the ground state, of a complex Hamiltonian is of great importance in quantum simulations. Many proposals have been introduced and experimentally realized, among which are quantum variational eigensolver and heat-bath algorithmic cooling, with the former hindered by local minima and the latter lacking of complex system Hamiltonians. Here we introduce a dissipative quantum-classical hybrid scheme, the probabilistic eigensolver. The scheme repeatedly uses an ancilla qubit to acquire information on the system, based on which it postselectively lowers the average energy of the system. The optimal reduction is achieved through classical optimization with a single variational parameter. We describe the implementation of the probabilistic eigensolver with trapped-ion systems and demonstrate the performance by numerically simulating the ground-state preparation of several paradigmatic models, including the Rabi and the Hubbard models. We believe the scheme would enrich the functionalities of universal quantum simulators and be useful as a module for various quantum-computation tasks.
\end{abstract}

DOI: 10.1103/PhysRevA.101.052333

\section{INTRODUCTION}

Simulating complex quantum systems is known to be an intractable task if accomplished with classical computing resources. This is because the dimension of the Hilbert space needed to describe a quantum system increases exponentially with its number of constituents. Quantum simulation [1], i.e., the use of a fully controlled quantum system that simulates the dynamics of another one, was originally proposed to overcome this problem, and has led to plenty of developments in different quantum platforms [2]. On the other hand, quantum computing is a more general approach that allows us not only to simulate quantum dynamics, but also to solve systems of linear equations [3], linear differential equations [4-6], or the eigenvalue problem of complex Hamiltonians [7,8], with an exponential speedup. Regarding the latter, it is important to remark that the knowledge of the energy spectrum of a system is crucial in different fields such as quantum chemistry and condensed-matter physics.

The phase estimation algorithm [7] is a prominent method for finding eigenvalues and preparing eigenstates of target Hamiltonians; however, other approaches have also been designed. Among them, we can mention the use of adiabatic evolution $[9,10]$ and variational quantum eigensolvers [11-13].

\footnotetext{
*zhangjn@baqis.ac.cn

†enr.solano@gmail.com
}

The adiabatic method starts from a simple Hamiltonian and slowly evolves to a complex Hamiltonian of interest. During the evolution the adiabaticity condition has to be fulfilled. This can be challenging, especially when critical points corresponding to quantum phase transitions are present. Here, the energy gap between two or more eigenstates becomes exponentially small as the size of the system increases. On the other hand, variational eigensolvers are designed to prepare the ground state (or excited state [11,12]) of a complex Hamiltonian, which interestingly combine a quantum processor and a classical optimization algorithm. The variational eigensolvers have been implemented experimentally in state-of-theart quantum platforms such as photonics [11], superconducting circuits [14], or trapped ions [15,16], and their accuracy in eigenstates preparation, as well as in the computing of the associated eigenvalues, highly depends on the flexibility of the ansatz $[14,15,17]$. In this respect, having a more complex and flexible input state, or ansatz, implies that more variational parameters have to be optimized simultaneously, which could be hinderedby the presence of local minima. In addition, the preparation of the ansatz may involve nontrivial entanglement operations among the available quantum registers. Thus, it is important to explore alternative algorithms to be applied in near-future quantum processors [18].

State preparation can also be achieved by dissipatively pumping entropy into the environment. For example, heatbath algorithmic cooling [19,20], which is widely used in nuclear magnetic resonance quantum computing, transfers 
entropy from the system to ancillary spins that rapidly thermalize through the environment. This method is usually used for preparing the spin system in a fully polarized product state. Dissipative methods to prepare entangled states also exist [21], with proof-of-principle experiments performed in trapped ions [22,23] and superconducting circuits [24]. Although powerful and robust, these state-oriented proposals assume complete prior information of the target state and thus cannot be used to find solutions encoded in some complex Hamiltonians. A closely related proposal, called dissipative quantum computation [25], designs a dissipative process whose unique steady state is the ground state of the target Hamiltonian; however, it only works for frustration-free systems.

In this article, we propose a hybrid classical-quantum eigensolver that uses projective measurements on a single ancilla qubit to probabilistically prepare the eigenstates of general multipartite Hamiltonians. Unlike the quantum variational solver or adiabatic state preparation, the only requirement in our method is that the initial state has a non-negligible overlap with the desired target state. Previously, there were theoretical attempts on probabilistic preparation of the ground state of a single qubit [26] or an $n$-level quantum system [27-29], with the former experimentally demonstrated with quantum optics [26]. Although the mechanism of our probabilistic eigensolver relies on arguments similar to those of Refs. [26-29], here we consider the implementation of this method in trapped-ion setups, which are capable of realizing various Hamiltonians consisting of multiple qubits and harmonic motional modes. In addition, our proposed eigensolver is featured by the introduction of a classical optimization module to reduce the coherent circuit depth. Moreover, we extend the method to prepare excited states with minor modifications. In Sec. II, we introduce the general method for preparing an arbitrary target eigenstate, given that the overlap between the target and the initial state is nonzero. Afterwards, we present how the method can be combined with a classical optimization algorithm, resulting in a significant reduction on the number of steps required for the preparation, while maintaining acceptable success probability. In Sec. III, we present the toolkit that trapped-ion quantum simulators offer to implement our method. Finally, in Sec. IV, we present some models in which our probabilistic eigenstate preparation could be applied by using a trapped-ion quantum processor and demonstrate its performance with numerical simulations.

\section{GENERAL FRAMEWORK}

Consider a target quantum system described by the Hamiltonian $\hat{H}$ such that

$$
\hat{H}|j\rangle=E_{j}|j\rangle .
$$

Here, $|j\rangle\left(E_{j}\right)$ is the $j$ th eigenstate (eigenenergy) and the energy spectrum $\left\{E_{j}\right\}$ is bounded from below and sorted in ascending order. To prepare one of the eigenstates of $\hat{H}$, we consider a quantum system isomorphic to the target system and an additional ancillary qubit. The evolution of the whole system is described by a unitary operator acting on the product Hilbert space $\mathcal{H}_{\mathrm{S}} \otimes \mathcal{H}_{\mathrm{A}}$, with $\mathcal{H}_{\mathrm{S}}\left(\mathcal{H}_{\mathrm{A}}\right)$ being the Hilbert space of the target system (the ancilla qubit).
For preparing the $j$ th eigenstate $|j\rangle$, our method requires knowledge of all eigenenergies below $E_{j}$. As, in general, this is not the case, we have to start with the ground-state preparation. In this manner, this first step can be considered as a cooling protocol. To this end, we apply the following unitary operator on the whole system:

$$
\hat{W}_{\gamma}(\tau)=\exp \left[-i\left(\hat{H}_{\mathrm{S}}+\gamma\right) \hat{\sigma}_{\mathrm{A}}^{\mathrm{x}} \tau\right] .
$$

Here, $\hat{\sigma}_{\mathrm{A}}^{\mathrm{x}}$ is the $\mathrm{x}$ Pauli matrix acting on the ancillary qubit and the parameters $\gamma$ and $\tau$ are real numbers to be determined. $\hat{W}_{\gamma}(\tau)$ can be viewed as an evolution operator of the whole system with $\tau$ being the effective evolution time. We use $\gamma$ to shift the energy spectrum of $\hat{H}_{\mathrm{S}}$ such that $E_{0}+\gamma \geqslant 0$ (i.e., the shifted spectrum is always positive), where $E_{0}$ is the groundstate energy of the target system.

Our protocol is an iterative method where each repetition involves, first, initializing the ancillary qubit to a reference state $|0\rangle_{\mathrm{A}}$ such that $\hat{\sigma}_{\mathrm{A}}^{z}|0\rangle_{\mathrm{A}}=-|0\rangle_{\mathrm{A}}$, and second, applying $\hat{W}_{\gamma}(\tau)$. In the $k$ th iteration we obtain the state $\left|\Psi_{k}\right\rangle$, which is

$$
\begin{aligned}
\left|\Psi_{k}\right\rangle & =\hat{W}_{\gamma}(\tau)\left|\psi_{k-1}\right\rangle|0\rangle_{\mathrm{A}} \\
& =\hat{\mathcal{C}}_{\gamma}(\tau)\left|\psi_{k-1}\right\rangle|0\rangle_{\mathrm{A}}-i \hat{\mathcal{S}}_{\gamma}(\tau)\left|\psi_{k-1}\right\rangle|1\rangle_{\mathrm{A}} .
\end{aligned}
$$

Here, $\left|\psi_{k-1}\right\rangle$ is the normalized state vector in $\mathcal{H}_{\mathrm{S}}$ that results from the $k-1$ iteration, and the nonunitary operators are defined as $\hat{\mathcal{C}}_{\gamma}(\tau)=\cos [(\hat{H}+\gamma) \tau]$ and $\hat{\mathcal{S}}_{\gamma}(\tau)=$ $\sin [(\hat{H}+\gamma) \tau]$. Finally, we perform a projective measurement on the ancillary qubit and make postselection on the measurement results to select certain the output state in $\mathcal{H}_{\mathrm{S}}$. The two possible normalized output states, conditioned on the measurement results, are $\left|\psi_{k}\right\rangle_{0}=$ $\hat{\mathcal{C}}_{\gamma}(\tau) /\left(P_{k}^{0}\right)^{1 / 2}\left|\psi_{k-1}\right\rangle$ and $\left|\psi_{k}\right\rangle_{1}=\hat{\mathcal{S}}_{\gamma}(\tau) /\left(P_{k}^{1}\right)^{1 / 2}\left|\psi_{k-1}\right\rangle$, and their probabilities are $P_{k}^{0}=\sum_{j}\left|\left\langle j \mid \psi_{k-1}\right\rangle\right|^{2} \cos ^{2}\left[\left(E_{j}+\gamma\right) \tau\right]$ and $P_{k}^{1}=\sum_{j}\left|\left\langle j \mid \psi_{k-1}\right\rangle\right|^{2} \sin ^{2}\left[\left(E_{j}+\gamma\right) \tau\right]$, respectively. We would like to point out that, although we use a pure input state $\left|\psi_{k-1}\right\rangle$ to illustrate the process, our method can be straightforwardly applied to mixed input states without any modification.

Now, if we compare the average energy of the two possible output states with the average energy of the input state, we find that, for positive and small $\tau$, the following relation holds (see Appendix A):

$$
\langle\hat{H}\rangle_{k}^{(0)} \leqslant\langle\hat{H}\rangle_{k-1}^{(0)}<\langle\hat{H}\rangle_{k}^{(1)} .
$$

Here $\langle\cdot\rangle_{k}^{(i)} \equiv\left\langle\psi_{k}^{(i)}|\hat{H}| \psi_{k}^{(i)}\right\rangle$ and the superscript $i=0,1$ denotes the outcome of the projective measurement on the ancillary qubit. In other words, the average energy of the system is lowered compared with the one of the input state, i.e., the postselected state from the previous round, if we successfully project the ancillary qubit in $|0\rangle_{\mathrm{A}}$. Here, it is noteworthy to comment that the probability of projecting on the reference ancilla state $|0\rangle_{\mathrm{A}}$ (i.e., on the state that leads to an effective average energy reduction) is close to one for a sufficiently small $\tau$ (note that $P_{k}^{0}=1$ if $\tau=0$ ). An intuitive way to understand this energy reduction for small $\tau$ is provided when studying the transformation of the state. If $\left|\psi_{k-1}\right\rangle=\sum_{j} c_{j}^{k-1}|j\rangle$ is the system input state before the $k$ th iteration, the output state after the application of $\hat{W}_{\gamma}(\tau)$ and 
postselection of $|0\rangle_{\mathrm{A}}$ will be $\left|\psi_{k}\right\rangle=\left|\psi_{k}\right\rangle_{0} \equiv \sum_{j} c_{j}^{k}|j\rangle$, where the probability distribution of the state has changed as

$$
\left|c_{j}^{k}\right|^{2}=\frac{1}{P_{k}^{0}} \cos ^{2}\left[\left(E_{j}+\gamma\right) \tau\right]\left|c_{j}^{k-1}\right|^{2} .
$$

As $\cos ^{2}(\cdot)$ is a monotonically decreasing function in the vicinity of $\tau=0$, the probability amplitudes of those eigenstates with lower eigenenergies $E_{j}$ will be enhanced [note that a larger value of $E_{j}$ implies a lower value for the corresponding $\cos ^{2}(\cdot)$ function] compared with those with higher eigenenergies, leading to the decrease of the average energy - an effective cooling effect.

After the $k$ th iteration, we have to measure the average energy of the resulting state $\bar{E}^{k} \equiv\langle\hat{H}\rangle_{k}^{(0)}$, and compare it with the average energy of the previous stage $\bar{E}^{k-1}$. If $\mid \bar{E}^{k-1}-$ $\bar{E}^{k} \mid<\epsilon$ holds (with $\epsilon$ being a small positive value), we conclude that that the ground-state within a certain precision $\epsilon$ has been reached and stop the protocol. If the state does not converge at the $k$ th iteration, saying $\left|\bar{E}^{k-1}-\bar{E}^{k}\right|>\epsilon$, the output state $\left|\psi_{k}\right\rangle_{0}$ will be sent through another iteration to further reduce its energy.

An important feature of our protocol is that, if the initial state $\left|\psi_{0}\right\rangle$ has no overlap with the desired eigenstate, it is not possible to prepare it. It can be seen from Eq. (5) that, if the $(k-1)$ st input state has no overlap with a given eigenstate $|j\rangle$ (i.e., $c_{j}^{k-1}=0$ ), the resulting $k$ th state will also share the same condition $c_{j}^{k}=0$. Although this may seem a disadvantage, it can actually be used to prepare excited eigenstates. To this end, we start from an arbitrary initial state $\left|\phi_{0}\right\rangle$ and apply the unitary operation

$$
\hat{U}_{s}=\exp \left[-i\left(\frac{\pi}{2 E_{s}}\right) \hat{H} \hat{\sigma}_{\mathrm{A}}^{\mathrm{x}}\right]
$$

and measure the ancilla qubit. The action of the unitary operator $\hat{U}_{s}$ on a general state $|\phi\rangle$ is to conditionally produce a state $\left|\psi^{(0)}\right\rangle$ where the contribution of the basis state $|s\rangle$ goes to zero $\left(\left\langle s \mid \psi^{(0)}\right\rangle=0\right)$ as long as the ancilla qubit is projected on $|0\rangle_{\mathrm{A}}$. As a result, the output state after postselection has no overlap with the eigenstate $|s\rangle$. For example, to prepare the first-excited state of $\hat{H}$ starting from an arbitrary initial state $\left|\phi_{0}\right\rangle$, one starts by applying the unitary $\hat{U}_{0}$ and projecting the ancilla on the $|0\rangle_{\mathrm{A}}$ state. If the projection succeeds, one obtains a state $\left|\psi_{0}\right\rangle$ that has zero overlap with the ground state of $\hat{H}$, which serves as input state for the cooling stage. Then, one applies the previously described protocol (see Fig. 1), until the state converges to the lowest energy eigenstate that has a nonzero overlap with the initial state, which in this case is the first-excited state.

To prepare an arbitrary eigenstate $|j\rangle$ starting from $\left|\phi_{0}\right\rangle$, one first removes any overlap with lower-energy eigenstates by sequentially applying $\hat{U}_{j^{\prime}}$ and the following projective measurement with $j^{\prime}=0,1, \ldots, j-1$. If all of the postselection operations succeed, the resulting state is sent to the cooling stage, which would probabilistically converge to the desired eigenstate $|j\rangle$. Note that once a postselection operation fails, the whole procedure should be started all over again.

We consider now two different variants for the cooling stage, namely, the fixed-step and variational approaches.

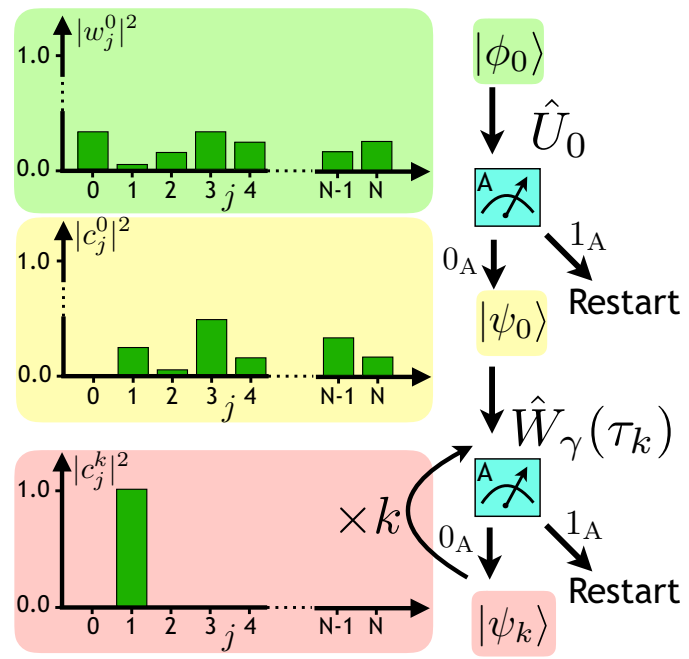

FIG. 1. Scheme for preparing the first-excited state of an $N$ dimensional Hamiltonian. First, we apply $\hat{U}_{0}$ and measure the ancilla qubit. Then we use postselection to remove the ground-state contribution from the initial state $\left|\phi_{0}\right\rangle$. After that, we apply the cooling unitary $\hat{W}_{\gamma}\left(\tau_{k}\right)$ and subsequent ancilla measurement $k$ times, until we reach convergence.

The fixed-step approach treats $\tau$ as a small fixed parameter. The operational procedure is as follows: After each implementation of $\hat{W}_{\gamma}(\tau)$, we perform a projective measurement on the ancilla qubit. We continue with the next iteration if the outcome is $|0\rangle_{\mathrm{A}}$, otherwise we restart the whole process. At the $k$ th stage of the process, we estimate the average energy of the output state $\bar{E}^{k}$. The process stops if $\left|\bar{E}^{k-1}-\bar{E}^{k}\right| \leqslant \epsilon$, where the precision $\epsilon$ is a small positive value. Otherwise, we continue the protocol by moving to the $(k+1)$ st stage.

In the variational approach, the value of the parameter $\tau$ is optimized in each stage such that it minimizes the average energy. This hybrid optimization is done by feeding the average energy obtained from the quantum platform to a classical optimization algorithm, which provides new trial value for $\tau$ to the quantum platform based on the given information (see Fig. 2). More specifically, the classical algorithm uses Brent's method to find a minimum in the interval $0<\tau_{k}<1$. After several optimization steps (trial steps), the classical algorithm will converge to $\tau_{k}$, the optimal value of $\tau$ at the $k$ th stage, and the quantum platform will estimate the average energy $\bar{E}^{k}$. As before, the process stops if $\left|\bar{E}^{k-1}-\bar{E}^{k}\right| \leqslant \epsilon$. At the end, we obtain a set of optimized values $\left\{\tau_{1}, \tau_{2}, \ldots, \tau_{k}\right\}$. Compared to the fixed-step approach, the variational approach allows the probabilistic preparation of the desired eigenstate with a substantial reduction on the circuit depth, i.e., with a qualitatively fewer gates and postselections.

Both the fixed-step and the variational approaches are probabilistic because we need to restart the whole procedure depending on the results of the projective measurements on the ancilla qubit and provide an estimation of the eigenenergy of the target eigenstate within the preset precision $\epsilon$. However, the variational protocol additionally provides a recipe for shallow-depth probabilistic preparation of the target state, as described by the set of optimized parameters $\left\{\tau_{1}, \ldots, \tau_{k}\right\}$. The overall success probability $P_{\text {suc }}$ of both protocols is ultimately 


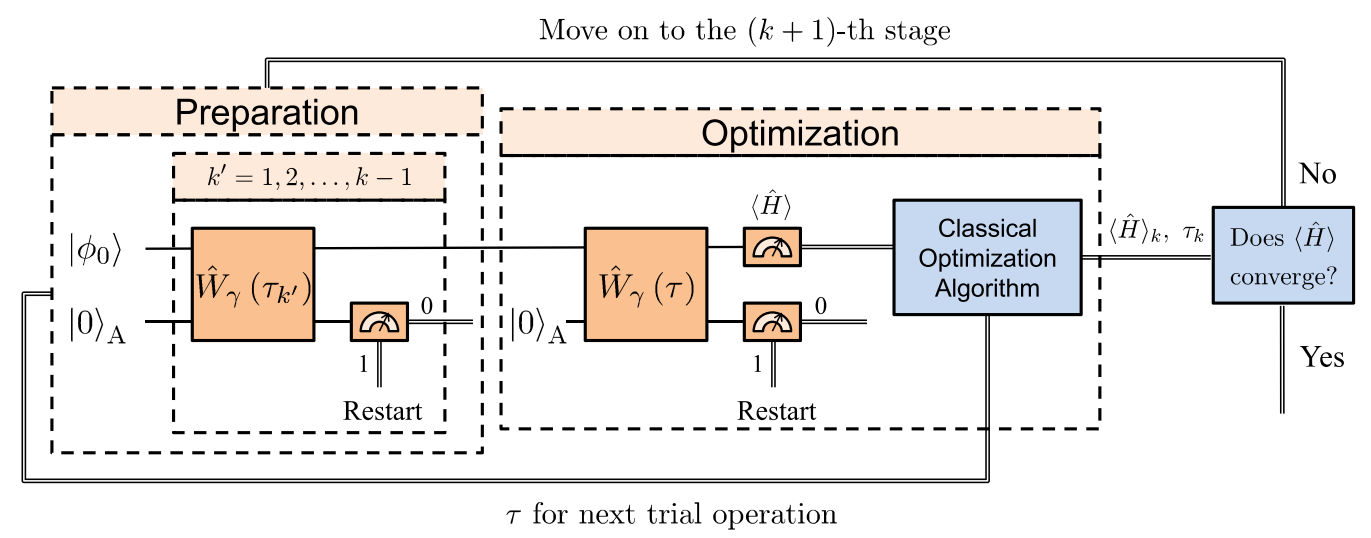

FIG. 2. Scheme for obtaining the optimal value of $\tau$ for the $k$ th stage in the variational approach to the probabilistic eigensolver. First, prepare the state at the $(k-1)$ st stage by applying the effective cooling $k-1$ times with optimized parameters $\left\{\tau_{1}, \tau_{2}, \ldots, \tau_{k-1}\right\}$. Then, search for the optimal value $\tau_{k}$ with the help of a classical optimization algorithm. Specifically, the quantum simulator estimates the average energy and feeds it into the classical algorithm, which determines whether the optimal value is reached or alternatively the next trial value for $\tau$.

bounded by the overlap between the initial state and the desired eigenstate, i.e., $P_{\text {suc }} \leqslant\left|\left\langle\psi_{0} \mid j^{\prime}\right\rangle\right|^{2}$. On the other hand, to maximize $P_{\text {suc }}$ for the preparation of eigenstate $\left|j^{\prime}\right\rangle$, one should choose the value of $\gamma$ to be the closest possible to $-E_{j^{\prime}}$. However, it is possible that we do not know the exact value of $E_{j^{\prime}}$, in which case one can always choose $\gamma$ to be minus the lower bound of the norm of the Hamiltonian, which can be analytically approximated in general.

Finally, we comment that the unitary operator $\hat{W}_{\gamma}(\tau)$ required in our method can be decomposed as $\hat{W}_{\gamma}(\tau) \equiv$ $\hat{W}(\tau) R_{\mathrm{x}}^{\mathrm{A}}(2 \gamma \tau)$, with $\hat{W}(\tau)=\exp \left(-\hat{H} \hat{\sigma}_{\mathrm{A}}^{\mathrm{x}} \tau\right)$ and $R_{\alpha}^{\mathrm{A}}(\theta) \equiv$ $\exp \left(-\frac{i \theta}{2} \hat{\sigma}_{\mathrm{A}}^{\alpha}\right), \alpha=\mathrm{x}, \mathrm{y}, \mathrm{z}$, being the single qubit rotation acting on the ancilla qubit. Now, the $\hat{W}(\tau)$ operator that entangles the system and the ancilla qubit, can be trotterized into a set of basic operations [30]. More specifically, suppose that the system Hamiltonian is composed of $M$ components $\hat{H}_{m}$ that do not commute with each other, namely $\hat{H}=\sum_{m=1}^{M} \hat{H}_{m}$. Then, the unitary evolution in Eq. (2) can be approximately written by using the second-order Trotter-Suzuki expansion,

$$
\begin{aligned}
\hat{W}(\tau)= & {\left[\hat{W}_{1}\left(\frac{\tau}{2 r}\right) \cdots \hat{W}_{M-1}\left(\frac{\tau}{2 r}\right) \hat{W}_{M}\left(\frac{\tau}{r}\right)\right.} \\
& \left.\times \hat{W}_{M-1}\left(\frac{\tau}{2 r}\right) \cdots \hat{W}_{1}\left(\frac{\tau}{2 r}\right)\right]^{r}+O\left(\frac{\tau^{3}}{r^{2}}\right),
\end{aligned}
$$

where $r$ is the number of Trotter steps and $\hat{W}_{m}(\tau)=$ $\exp \left(-i \hat{H}_{m} \hat{\sigma}_{\mathrm{A}}^{\mathrm{x}} \tau\right)$ with $m=1,2, \ldots, M$. Note that, by increasing the number of Trotter steps, arbitrary precision can be achieved in principle.

\section{TOOLKIT IN TRAPPED-ION PLATFORMS}

Trapped ions have been demonstrated to be suitable for digital [31] and analog [32,33] quantum simulations, having access not only to the manipulation of the ion-qubits, but also to the coherent control of their collective vibrational modes [34]. The digital-analog approaches which combine both resources could enhance the computational power of the quantum platform for solving hard problems [35], for example, nontrivial many-body models or even problems in quantum field theories [36].
We envision four types of primitive system-ancilla couplings in the toolkit of trapped-ion systems, including (1) $\hat{\sigma}_{i}^{\mathrm{X}} \hat{\sigma}_{\mathrm{A}}^{\mathrm{x}}$, (2) $\hat{a}_{m}^{\dagger} \hat{a}_{m} \hat{\sigma}_{\mathrm{A}}^{\mathrm{x}}$, (3) $\hat{\sigma}_{i_{1}}^{\mathrm{X}} \hat{\sigma}_{i_{i} \mathrm{O}}^{\mathrm{X}} \hat{\mathrm{A}}_{\mathrm{A}}^{\mathrm{x}}$, and (4) $\left(\hat{a}_{m}^{\dagger}+\hat{a}_{m}\right) \hat{\sigma}_{i}^{\mathrm{X}} \hat{\sigma}_{\mathrm{A}}^{\mathrm{X}}$. Using these interactions and the measurement on the ancilla, the protocol described in the previous section could be implemented in trapped-ion systems for a wide variety of models.

The free Hamiltonian $\hat{H}_{0}$ of an array of $N$ ions trapped in a linear Paul trap is written as follows:

$$
\hat{H}_{0}=\sum_{n=1}^{N} \frac{\hbar \omega_{0}}{2} \hat{\sigma}_{n}^{\mathrm{z}}+\sum_{m=1}^{N} \hbar \omega_{m}\left(\hat{a}_{m}^{\dagger} \hat{a}_{m}+\frac{1}{2}\right),
$$

where $\hat{\sigma}_{n}^{\mathrm{x}, \mathrm{y}, \mathrm{z}}$ are the Pauli matrices acting on the $n$th ion with $\omega_{0}$ being the frequency splitting of the two involved internal levels, and $\hat{a}_{m}\left(\hat{a}_{m}^{\dagger}\right)$ is the annihilation (creation) operator of the $m$ th collective motional mode with the mode frequency $\omega_{m}$. For a better readability, from now on we omit the subscript $n(m)$ of $\hat{\sigma}_{n}^{\mathrm{X}, \mathrm{y}, \mathrm{z}}\left(\hat{a}_{m}\right)$ when there is no ambiguity.

In the following, we provide a brief explanation of how to engineer the four types of system-ancilla couplings in a trapped-ion quantum simulator:

First, spin-spin couplings can be implemented by the Mølmer-Sørensen (MS) type of interaction, which takes the form of $\sigma_{i}^{\mathrm{x}} \sigma_{j}^{\mathrm{x}}$ and can be engineered between any pair of ions embedded in a linear chain of ions [37]. With the appropriate single qubit rotations on the first qubit, we can extend the coupling to the form $\hat{\sigma}^{\mathbf{n}} \sigma_{A}^{\mathrm{x}}$, which would correspond to a simulated local spin- $1 / 2$ operator $\hat{H}_{\mathrm{S}} \propto \hat{\sigma}_{\mathbf{n}}$, where $\mathbf{n}=$ $\left(n_{\mathrm{x}}, n_{\mathrm{y}}, n_{\mathrm{z}}\right)^{\mathrm{T}}$ is a unit vector.

Second, the $\hat{a}^{\dagger} \hat{a} \hat{\sigma}_{\mathrm{A}}^{\mathrm{x}}$ interaction term involving the $m$ th mode could be realized with a detuned red-sideband coupling, for which the Hamiltonian is written, in the rotating frame with respect $\hat{H}_{0}$ and after the rotating wave approximation, as

$$
\begin{aligned}
\hat{H}_{\mathrm{I}}= & \frac{\hbar \Omega_{\mathrm{A}}}{2}\left[\left(\hat{\sigma}^{+} e^{i \Delta t}+\hat{\sigma}^{-} e^{-i \Delta t}\right)\right] \\
& +\frac{i \hbar \eta \Omega_{\mathrm{A}}}{2}\left(\hat{a} \hat{\sigma}^{+} e^{-i \delta t}-\hat{a}^{\dagger} \hat{\sigma}^{-} e^{i \delta t}\right),
\end{aligned}
$$

where $\Omega_{\mathrm{A}}$ is the ancilla Rabi frequency, $\eta \equiv \eta_{m}^{\mathrm{A}}=$ $\delta k \sqrt{\hbar /\left(2 M \omega_{m}\right)}$ is the Lamb-Dicke (LD) parameter associated with the $m$ th mode and the ancilla qubit, with $\delta k$ being the 
(a)

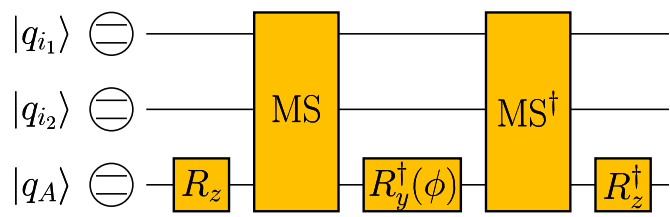

(b)

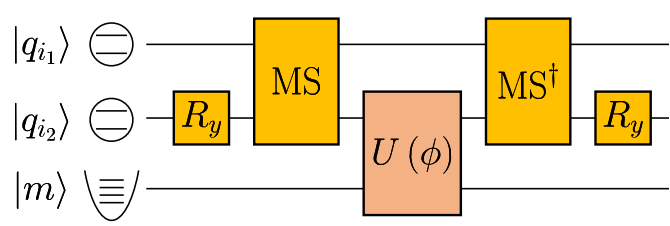

FIG. 3. Quantum circuits for the trapped-ion toolkit. (a) Scheme for implementing the unitary $\exp \left(-\frac{i \phi}{2} \hat{\sigma}_{i_{1}}^{\mathrm{X}} \hat{\sigma}_{i_{2}}^{\mathrm{x}} \hat{\sigma}_{\mathrm{A}}^{\mathrm{X}}\right)$ by using single-qubit rotations and global Mølmer-Sørensen gates MS $=\exp \left(-\frac{i \pi}{4} \sum_{i<j} \hat{\sigma}_{i}^{x} \hat{\sigma}_{j}^{x}\right)$, where the summation runs over all possible pairs of qubits. Here, the single-qubit rotations are defined as $R_{\alpha}(\phi)=\exp \left(-\frac{i \phi}{2} \hat{\sigma}^{\alpha}\right)$ with $\alpha=x, y$, or $z$ and we use $R_{\alpha}\left(\frac{\pi}{2}\right) \equiv R_{\alpha}$ for simplicity. (b) Scheme for implementing the unitary operator $\exp \left[-\frac{i \phi}{2}\left(\hat{a}+\hat{a}^{\dagger}\right) \hat{\sigma}_{i}^{\mathrm{x}} \hat{\sigma}_{\mathrm{A}}^{\mathrm{y}}\right]$ using MS gates, single-qubit gates, and the analog block $\hat{U}_{\mathrm{R}}(\phi)$ involving the $m$ th motional mode.

transferred wave vector of the laser and $M$ being the mass of the ion, and $\Delta$ and $\delta$ are the detunings to the carrier and the first red-sideband transitions, respectively. In the dispersive regime, i.e., $\Omega_{\mathrm{A}} \ll \Delta$ and $\eta \Omega_{\mathrm{A}} \ll \delta$, the effective Hamiltonian can be written as follows [38]:

$$
\hat{H}_{\mathrm{eff}}=\frac{\hbar \Omega_{\mathrm{A}}^{2}}{4 \Delta} \hat{\sigma}^{\mathrm{Z}}+\frac{\hbar \eta^{2} \Omega_{\mathrm{A}}^{2}}{4 \delta}\left(\hat{a}^{\dagger} \hat{a}+\frac{1}{2}\right) \hat{\sigma}^{\mathrm{z}}
$$

where the AC-Stark shift term can be absorbed into the free Hamiltonian, and the term that depends on the bosonic operators can be transformed into $\hat{a}^{\dagger} \hat{a} \hat{\sigma}_{\mathrm{A}}^{\mathrm{x}}$ by appropriate single-qubit rotations on the ancilla.

The third term $\hat{\sigma}_{1}^{z} \hat{\sigma}_{2}^{z} \hat{\sigma}_{3}^{z}$ can be implemented by combining the controlled-not (CNOT) gates [8] or the MS gates [39] with single-qubit $z$ rotations. Moreover, global MS gates [40] can be used to reduce the number of CNOTs or two-qubit MS gates, increasing the process fidelity. Figure 3(a) shows a recipe to construct this nonlocal spin operation with global MS gates.

Finally, similarly to the previous quantum circuit, we show that the dynamics governed by $\left(\hat{a}^{\dagger}+\hat{a}\right) \hat{\sigma}_{i}^{\mathrm{x}} \hat{\sigma}_{\mathrm{A}}^{\mathrm{X}}$ can be implemented by combining CNOT gates (or MS gates [41]) with analog blocks that involve the dipolar coupling between the $m$ th motional mode with the ancilla qubit. In Fig. 3(b) we show the complete circuit to achieve the desired interaction. The central part of the circuit is a unitary operator produced by a simulated dipolar coupling that can be implemented by simultaneously driving the red-sideband and blue-sideband transitions of the ancilla qubit [42]. The associated evolution operator $\hat{U}_{\mathrm{R}}(\phi)$ in the rotating frame defined by the free Hamiltonian $\hat{H}_{0}$ is written as follows:

$$
\hat{U}_{\mathrm{R}}(\phi)=\exp \left[-\frac{i \phi}{2}\left(\hat{a}+\hat{a}^{\dagger}\right) \hat{\sigma}_{\mathrm{A}}^{\mathrm{x}}\right],
$$

where $\phi$ is proportional to the Rabi frequency $\Omega_{\mathrm{A}}$, the LambDicke parameter $\eta_{m}^{\mathrm{A}}$, and the interaction time. Another qubit will be involved by a pair of CNOT gates and all the other single-qubit rotations are used for changing the basis of the Pauli matrices.

With the described toolkit available on trapped-ion quantum platforms, the probabilistic eigensolver can be extended to a wide range of models involving both qubits and bosonic modes. The latter include spin-spin interaction models like the Ising, $X Y$, or Heisenberg models [43], quantum Rabi models [44-47], the Dicke models [48], or second-quantization Hamiltonians like the Holstein model [49]. Also, more general methods such as those for computing $n$-time correlation functions [50] or to simulate dissipative processes [51] could benefit from the presented eigensolver as the latter can be used to prepare arbitrary eigenstates. In the next section, we numerically investigate the performance of the probabilistic eigensolver with several examples, including a single harmonic oscillator, the quantum Rabi model, and the Hubbard model.

\section{EXAMPLES}

\section{A. Harmonic oscillator}

One of the simplest and most important models of quantum mechanics is the quantum harmonic oscillator, which gives a mathematical description of various physical phenomena, including mechanical oscillators in harmonic potentials and electromagnetic fields [52]. The Hamiltonian of a single harmonic oscillator is written as follows:

$$
\hat{H}=\hbar \omega_{\text {h.o. }} \hat{a}^{\dagger} \hat{a},
$$

where $\hat{a}\left(\hat{a}^{\dagger}\right)$ is the annihilation (creation) operator of the mode with the mode angular frequency $\omega_{\text {h.o. }}$.

The Hamiltonian in Eq. (12) provides an equal-spacing spectrum with an infinite number of levels. One could think on a simple example of the protocol presented in Sec. II, where the application of a unitary of the form $\hat{W}(\tau)=$ $\exp \left[-i \tau \hat{a}^{\dagger} \hat{a} \hat{\sigma}_{\mathrm{A}}^{\mathrm{X}}\right]$ (see Sec. III) in a single ion, combined with measurements on the ancilla, could lead to a probabilistic preparation of the motional ground state. Note that the ground-state energy for a harmonic oscillator is positive, thus $\gamma$ is set to zero. Starting from, for example, a thermal state, each time the ancilla is measured to be in the state $|0\rangle_{\mathrm{A}}$ after applying $\hat{W}(\tau)$, the motional state is effectively "cooled down" (i.e., the average energy is lowered).

In Fig. 4, we show the results for the probabilistic groundstate preparation of a harmonic oscillator with $\omega_{\text {h.o. }}=1$. The initial state is naturally chosen as a thermal equilibrium state characterized by the thermal average number $\bar{n}_{\text {th }}=0.5$. Both the fixed-step cooling with $\tau=0.3$ [Fig. 4(a)] and the variational cooling [Fig. 4(b)] show a monotonically decreasing average energy $\langle\hat{H}\rangle$ with respect to the number of iterations $k$. The overall success probability $P_{\text {suc }}(k)$ up to the $k$ th stage is defined as

$$
P_{\mathrm{suc}}(k)=\prod_{k^{\prime}=1}^{k} P_{k^{\prime}}^{0},
$$

where $P_{k^{\prime}}^{0}$ is the probability of projecting the ancilla to $|0\rangle_{\mathrm{A}}$ at the $k^{\prime}$ th iteration. It is shown in Fig. 4 that both in the fixed-step and variational cases, $P_{\text {suc }}(k)$ decreases towards a saturate value around 0.65 . In this example, the fixed-step 

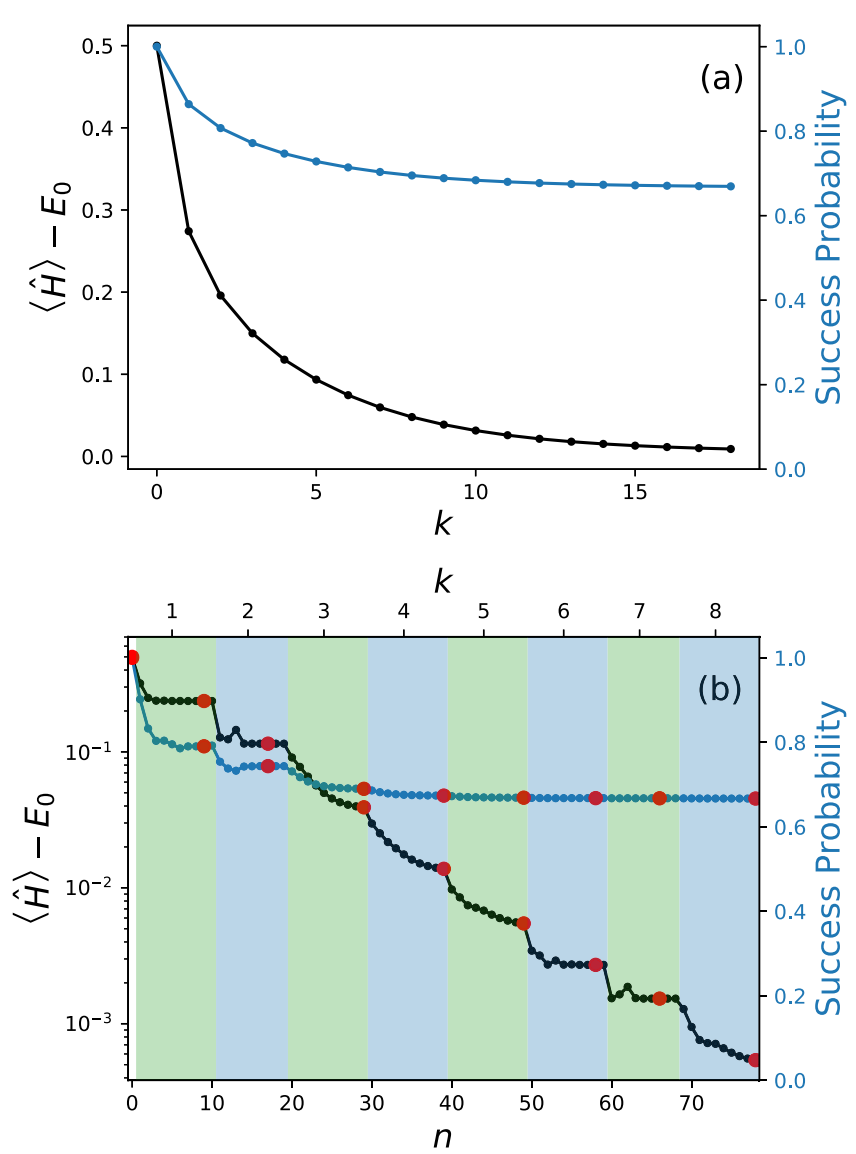

FIG. 4. Probabilistic ground-state preparation of a single harmonic mode. (a) Average energy $\langle\hat{H}\rangle$ (black dots) and the overall success probability $P_{\text {suc }}(k)$ (blue dots) as functions of the number of iterations $k$ for the fixed-step protocol. The normalized step size $\tau$ is fixed to be 0.3 . (b) Average energy $\langle\hat{H}\rangle$ (red dots over the black curve) and the overall success probability $P_{\text {suc }}(k)$ (red dots over the blue curve) as functions of the number of iterations $k$ for the optimized variational protocol. Black dots represent results for all the different trial steps that are needed until reaching convergence for the optimum $\tau_{k}$, which are $\{0.8487,0.5044,0.9919,0.9919,0.9910,0.7430,0.4194,0.9881\}$.

and the variational protocols reach convergence in 18 and 8 steps, respectively, where a precision of $\epsilon=10^{-3}$ is pursued. Figure 4(b) shows that the total number of trial steps (black dots) needed to obtain the optimized variational protocol are around 80 ( $\approx 10$ trials per step). However, once you have the set of optimized values $\left\{\tau_{k}\right\}$, the variational approach requires fewer steps than the fixed-step approach, which reduces the circuit depth and alleviates the requirement for long-term quantum coherence.

\section{B. Quantum Rabi model}

The quantum Rabi model [53] describes the dipolar interaction between a magnetic dipole and an oscillating magnetic field. Recently, its quantum simulation has been realized experimentally with highly tunable parameters in many physical platforms, including superconducting circuits [54,55] and trapped ions [56]. The Hamiltonian of the quantum Rabi model can be separated into two noncommuting parts,

$$
\hat{H}=\hat{H}_{1}+\hat{H}_{2} \text {, with }
$$

$$
\begin{aligned}
& \hat{H}_{1}=\frac{\hbar \omega_{0}}{2} \hat{\sigma}^{\mathrm{z}}+\hbar \omega \hat{a}^{\dagger} \hat{a}, \\
& \hat{H}_{2}=\hbar g\left(\hat{a}+\hat{a}^{\dagger}\right) \hat{\sigma}^{\mathrm{x}},
\end{aligned}
$$

where $\hat{\sigma}^{\mathrm{x}}$ and $\hat{\sigma}^{\mathrm{z}}$ are the Pauli matrices acting on the two-level system with the energy splitting $\omega_{0}, \hat{a}\left(\hat{a}^{\dagger}\right)$ is the annihilation (creation) operator of the harmonic mode with frequency $\omega$, and $g$ is the coupling strength of the dipolar interaction.

We implement $\hat{W}(\tau)$ through the second-order TrotterSuzuki expansion,

$$
\hat{W}_{\gamma}(\tau)=\left[\hat{W}_{1}\left(\frac{\delta \tau}{2}\right) \hat{W}_{2}(\delta \tau) \hat{W}_{1}\left(\frac{\delta \tau}{2}\right)\right]^{r}
$$

where $\delta \tau=\tau / r$ is the size of the Trotter step and $\hat{W}_{j}(\tau)=$ $\exp \left(-i \hat{H}_{j} \hat{\sigma}_{\mathrm{A}}^{\mathrm{x}} \tau\right)$ with $j=1$ and $2 . \hat{W}_{1}$ could be implemented by combining the first and second interactions described in Sec. III, while $\hat{W}_{2}$ requires the implementation of the fourth interaction. Overall, the simulation requires two qubits and a motional mode. Note that, depending on the step size $\tau$, more than one Trotter step may be needed.

Figure 5 shows the results for the ground-state preparation of the quantum Rabi model in the deep strong-coupling regime [57]. We choose the state $|\downarrow\rangle|0\rangle$ as the initial state, for it can be easily prepared by optical pumping and sideband cooling. Comparing both cases we clearly see how the variational approach offers a faster route to the ground-state preparation, with an overall success probability around $60 \%$, very similar to the fixed-step approach.

\section{Hubbard model}

The Hubbard model [58] is one of the most important models in solid-state physics. In spite of the simplicity in form, it describes the quantum phase transition between superconducting and Mott-insulator phases. The analytical solution has not been available for arbitrary dimensions and the exact numerical treatment is believed to be hard for classical computers because of the sign problem.

To illustrate the performance of our protocol in a system with only qubits, we consider the one-dimensional Hubbard model with $L$ sites and open boundary conditions, whose Hamiltonian is written as follows:

$$
\hat{H}=-t \sum_{i=1}^{L-1} \sum_{\sigma}\left(\hat{c}_{i, \sigma}^{\dagger} \hat{c}_{i+1, \sigma}+\text { H.c. }\right)+U \sum_{i=1}^{L} \hat{c}_{i, \uparrow}^{\dagger} \hat{c}_{i, \uparrow} \hat{c}_{i, \downarrow}^{\dagger} \hat{c}_{i, \downarrow},
$$

where $t$ and $U$ are the nearest-neighbor hopping strength and the on-site interaction strength, respectively. This model can be mapped to a nearest-neighbor spin chain of $2 N$ spins through the Jordan-Wigner transformation [59], which establishes a mapping between a fermionic operator and a set of spin operators. We choose the one-dimensional Hubbard model as an example, while the extension to two- and threedimensional (2D and 3D) models can be done in trapped ions by using nonlocal interactions [36]. The operator in Eq. (2) can be constructed by using the three-body spin operators described in Sec. III and the Suzuki-Trotter expansion. For 

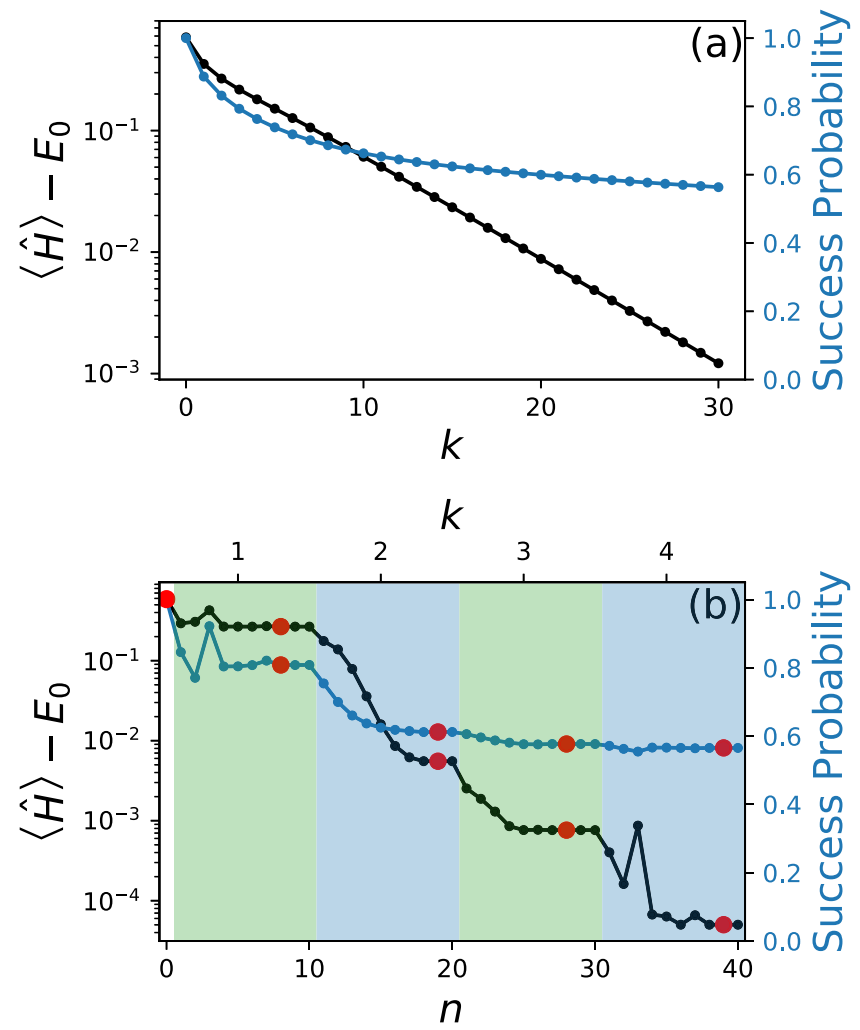

FIG. 5. Probabilistic ground-state preparation of the quantum Rabi model with $\omega_{0}=1.2, \omega=0.8$, and $g=1.0$. The energy is in units of $g$ and the number of stages is denoted by $k$. The number of Trotter steps is chosen as $r=3$. (a) Fixed-step probabilistic state preparation. The step size is chosen as $\tau_{\text {fix }}=0.3$. (b) Variational probabilistic state preparation. The total number of trial steps is denoted by $n$ (black dots). The trial steps for different stages are discriminated by different background colors. Red dots represent the energy and overall probability for different stages using the optimized $\tau$ values $\{0.4762,0.9839,0.9032,0.5575\}$.

the simulations, we choose $t=1$ and $U=2$ if not explicitly specified otherwise.

Figure 6 shows the numerical simulations for the groundstate preparation of the two-site and three-site Hubbard models using the variational approach for probabilistic state preparation. As initial states, we choose $|\uparrow \downarrow \uparrow \downarrow\rangle$ and $|\downarrow \uparrow \downarrow \downarrow \uparrow \downarrow\rangle$ for the two-site and three-site models, respectively. In addition, the $\hat{W}_{\gamma}(\tau)$ operation is implemented by using the symmetric Trotter expansion with $r=3$ Trotter steps. The results show that, with less than 10 steps, one can obtain the ground state for both models. The overall success probability is in this case around $15 \%$ which is still an acceptable value.

\section{v. CONCLUSIONS}

We have presented a probabilistic eigensolver which is capable of preparing arbitrary eigenstates of Hamiltonians that are implementable with a trapped-ion quantum processor. The method, applicable to a digital or digital-analog quantum simulator, requires control operations and measurements on an extra ancilla qubit. Moreover, we provide a recipe to enhance the performance of the probabilistic eigenstate prepa-
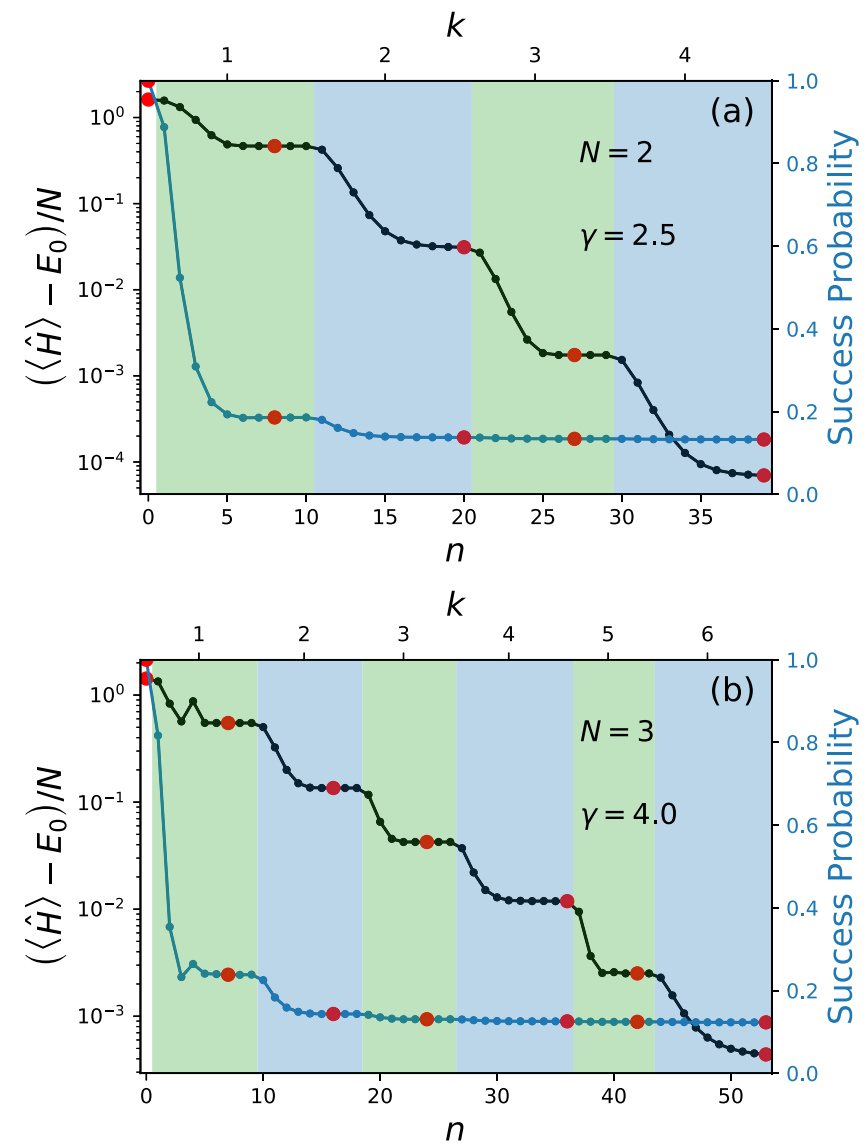

FIG. 6. Probabilistic ground-state preparation of (a) two-site and (b) three-site Hubbard models. The black dots indicate the average energy of the trial states after successfully projecting the ancilla qubit to the reference state $|0\rangle_{\mathrm{A}}$, while the blue dots represent the overall success probability. Red dots represent the average energy and overall success probability after the $k$ th stage, which is done by applying the $\hat{W}_{\gamma}(\tau)$ unitary with the optimized $\tau_{k}$, using $r=3$ Trotter steps. The optimized taus are $\{0.3692,0.3959,0.3684,0.3959\}$ and $\{0.2694,0.3661,0.3247,0.3973,0.2726,0.3959\}$ for the two-site and three-site models, respectively.

ration by means of a hybrid classical-quantum optimization algorithm. We describe a basic toolbox natural in trapped-ion quantum platforms, which can be used as building blocks to implement the method for complex Hamiltonian models. Finally, we numerically simulate the method for some interesting examples that could be implemented in state-of-the-art trapped-ion setups.

\section{ACKNOWLEDGMENTS}

This work was supported by the National Key Research and Development Program of China under Grants No. 2016YFA0301900 and No. 2016YFA0301901 and the National Natural Science Foundation of China Grants No. 11374178, No. 11574002, No. 11504197, and No. 11974200. We also acknowledge financial support from Spanish Government via PGC2018-095113-B-I00(MCIU/AEI/FEDER, UE), Basque Government via IT986-16, as well as from QMiCS (820505) and OpenSuperQ(820363) of the EU 
Flagship on Quantum Technologies, and the EU FET Open Grant Quromorphic. I.A. acknowledges support from Basque Government Ph.D. Grant No. PRE-2015-1-0394. J.C. acknowledges support from the UPV/EHU grant EHUrOPE.

\section{APPENDIX A: PROBABILISTIC COOLING EFFECT}

For an arbitrary input state $|\psi\rangle=\sum_{j} c_{j}|j\rangle$, the state for the system plus the ancilla qubit after applying $\hat{W}_{\gamma}(\tau)$ is denoted as $|\Psi\rangle \equiv \hat{W}_{\gamma}(\tau)|\psi\rangle\left|0_{\mathrm{A}}\right\rangle$. The values of the average energy with respect to the input state and conditioned output states are

$$
\begin{aligned}
\langle\hat{H}\rangle & =\left\langle\psi\left|\hat{H}_{\mathrm{S}}\right| \psi\right\rangle=\sum_{j} E_{j}\left|c_{j}\right|^{2}, \\
\langle\hat{H}\rangle^{(0)} & =\left\langle\Psi\left|\hat{H}_{\mathrm{S}} \otimes\right| 0_{\mathrm{A}}\right\rangle\left\langle 0_{\mathrm{A}}|| \Psi\right\rangle \\
& =\frac{1}{P_{0}} \sum_{j} E_{j}\left|c_{j}\right|^{2} \cos ^{2}\left[\left(E_{j}+\gamma\right) \tau\right], \\
\langle\hat{H}\rangle^{(1)} & =\left\langle\Psi\left|\hat{H}_{\mathrm{S}} \otimes\right| 1_{\mathrm{A}}\right\rangle\left\langle 1_{\mathrm{A}}|| \Psi\right\rangle \\
& =\frac{1}{P_{1}} \sum_{j} E_{j}\left|c_{j}\right|^{2} \sin ^{2}\left[\left(E_{j}+\gamma\right) \tau\right],
\end{aligned}
$$

where $P_{0}=\sum_{j}\left|c_{j}\right|^{2} \cos ^{2}\left[\left(E_{j}+\gamma\right) \tau\right]$ and $P_{1}=\sum_{j}\left|c_{j}\right|^{2}$ $\sin ^{2}\left[\left(E_{j}+\gamma\right) \tau\right]$. For small $\tau$, i.e., $\left(E_{j}+\gamma\right) \tau \ll 1$, we expand $\langle\hat{H}\rangle^{(0)}$ to the second order in $\tau$ and calculate the difference between $\langle\hat{H}\rangle^{(0)}$ and $\langle\hat{H}\rangle$ as follows:

$$
\left\langle\hat{H}_{\mathrm{S}}\right\rangle-\left\langle\hat{H}_{\mathrm{S}}\right\rangle^{(0)}=\sum_{j}\left(\left|c_{j}\right|^{2}-\left|c_{j}^{\prime}\right|^{2}\right) E_{j},
$$

where the new probability distribution is related with the old one as

$$
\begin{aligned}
\frac{c_{j}^{\prime}}{c_{j}} & =\frac{1}{P_{0}} \cos \left[\left(E_{j}+\gamma\right) \tau\right] \\
& \approx \frac{1}{P_{0}}\left[1-\frac{1}{2}\left(E_{j}+\gamma\right)^{2} \tau^{2}\right]+O\left(\tau^{4}\right) .
\end{aligned}
$$

Because $E_{0}+\gamma \geqslant 0$, the factor $1-\frac{1}{2}\left(E_{j}+\gamma\right)^{2} \tau^{2}$ decreases for higher excited states, meaning that the higher the energy of the state, the lower the probability amplitude at the output state. Similarly, we can calculate the difference between $\left\langle\hat{H}_{\mathrm{S}}\right\rangle^{(1)}$ and $\left\langle\hat{H}_{\mathrm{S}}\right\rangle$ to the leading order and obtain the following expression:

$$
\left\langle\hat{H}_{\mathrm{S}}\right\rangle^{(1)}-\left\langle\hat{H}_{\mathrm{S}}\right\rangle=\sum_{j}\left(\left|c_{j}^{\prime \prime}\right|^{2}-\left|c_{j}\right|^{2}\right) E_{j},
$$

where now the new probability distribution is related with the old one as

$$
\begin{aligned}
\frac{c_{j}^{\prime \prime}}{c_{j}} & =\frac{1}{P_{1}} \sin \left[\left(E_{j}+\gamma\right) \tau\right] \\
& \approx \frac{1}{P_{1}}\left(E_{j}+\gamma\right) \tau+O\left(\tau^{3}\right) .
\end{aligned}
$$

Notice that, in this case, the lower the energy of the state, the lower the ratio between the new and the old probability amplitude.

\section{APPENDIX B: SCALING ANALYSIS}

In this Appendix, we study the scaling behavior of the probabilistic eigensolver in the preparation of the ground state of a generic Hamiltonian. Specifically, we focus on the dependence of the number of cooling steps on the infidelity of the prepared ground state. To simplify the scenario, we analytically obtain the scaling behavior of the number of cooling steps in a cooling process with constant cooling strength, which serves as an upper bound for the number of cooling steps in a classically assisted cooling process with optimized cooling strength for each cooling step.

A generic input state is $\left|\psi_{\text {in }}\right\rangle=\sum_{j} c_{j}|j\rangle$. If there are $k$ steps and we get $k$ zeros, we get the following unnormalized state:

$$
\left|\psi_{\text {out }}\right\rangle=\sum_{j} c_{j} \cos ^{k} \phi_{j}|j\rangle
$$

with $\phi_{j}=E_{j} \tau+\gamma$. Let us define the condition that the state is $\epsilon$ convergent to the ground state as

$$
\frac{\sum_{j=1}^{M} P_{j} \cos ^{2 k} \phi_{j}}{P_{0} \cos ^{2 k} \phi_{0}} \leqslant \epsilon,
$$

with $P_{j} \equiv\left|c_{j}\right|^{2}$.

In the parameter regime that $0 \leqslant \phi_{0}<\phi_{1}<\cdots<\phi_{M} \leqslant$ $\frac{\pi}{2}$, thus $\cos \phi_{1} \geqslant \cos \phi_{j}$ for $j>2$ and it is evident that the following inequality is valid:

$$
\frac{\sum_{j=1}^{M} P_{j} \cos ^{2 k} \phi_{j}}{P_{0} \cos ^{2 k} \phi_{0}} \leqslant \frac{\left(1-P_{0}\right)}{P_{0}}\left(\frac{\cos \phi_{1}}{\cos \phi_{0}}\right)^{2 k},
$$

where we make use of the fact that, in the parameter regime, $1 \geqslant \cos \phi_{1}>\cos \phi_{2}>\cdots>\cos \phi_{M} \geqslant 0$. Comparing the right-hand sides of the above two equations, we obtain the scaling for the number of steps $k$ as follows:

$$
k \geqslant \frac{1}{2} \ln \frac{P_{0} \epsilon}{1-P_{0}} / \ln \frac{\cos \phi_{1}}{\cos \phi_{0}} .
$$

The denominator can be further processed as

$$
\begin{aligned}
\ln \frac{\cos \phi_{1}}{\cos \phi_{0}} & =\ln \left[1-\tan \phi_{0} \Delta \tau-\frac{1}{2} \Delta^{2} \tau^{2}+O\left(\Delta^{3} \tau^{3}\right)\right] \\
& =-\left(\tan \phi_{0} \Delta \tau+\frac{1}{2} \Delta^{2} \tau^{2}\right)+O\left(\Delta^{3} \tau^{3}\right),
\end{aligned}
$$

where $\Delta=E_{1}-E_{0}$ is the energy gap between the ground and the first-excited state. Finally, we obtain

$$
k \geqslant-\frac{1}{2} \ln \frac{P_{0} \epsilon}{1-P_{0}}\left(\tan \phi_{0} \Delta \tau+\frac{1}{2} \Delta^{2} \tau^{2}\right)^{-1} .
$$

\section{APPENDIX C: NOISE ANALYSIS}

All realistic quantum computations are subjected to noise, leading to decoherence. Here we study how dephasing or depolarizing errors can harness the presented cooling protocol.

First, we consider the case in which each qubit is subjected to a dephasing channel only while performing multiqubit gates. This simplified scenario is reasonable because dephasing affects more to multiqubit operations, as these are slower than single-qubit gates. Let us consider the quantum circuit in Fig. 3(a) as our ideal process. The quantum map 


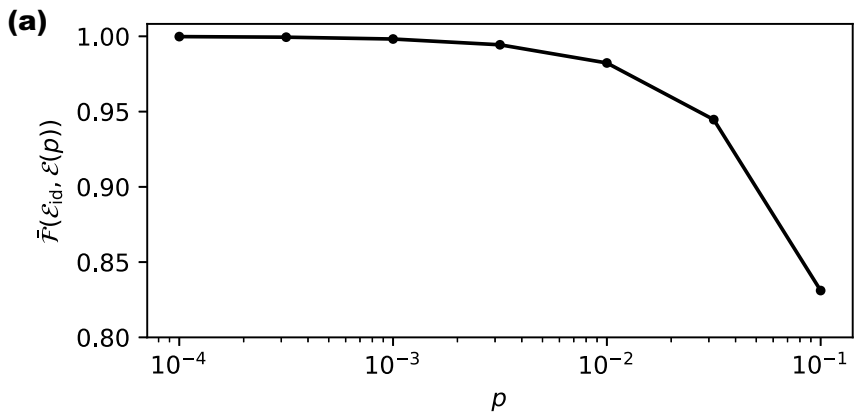

(b)

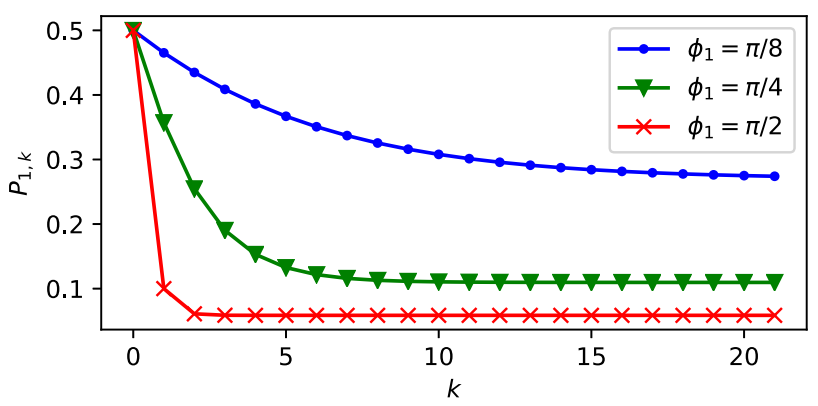

FIG. 7. Noisy analysis. (a) The average fidelity between the ideal and noisy processes for the quantum circuit in Fig. 3(a). The horizontal axis is the single-qubit dephasing rate per multiqubit gate. (b) The excited-state probability $P_{1, k}$ after $k$ successful steps of cooling, when the probabilistic eigensolver is subjected to a depolarizing environment characterized by the error rate $\delta=0.1$. The input state is chosen as the completely mixed state, and the cooling strength is characterized by $\phi_{1}=\pi / 8, \pi / 4$, and $\pi / 2$.

corresponding to the ideal process is

$$
\mathcal{E}_{\mathrm{id}}=\mathcal{U}_{R_{z}^{\dagger}} \circ \mathcal{U}_{\mathrm{MS}} \circ \mathcal{U}_{R_{y}^{\dagger}(\phi)} \circ \mathcal{U}_{\mathrm{MS}} \circ \mathcal{U}_{R_{z}},
$$

where $\circ$ means the concatenation of quantum maps, and the unitary channels are $\mathcal{U}_{O}(\rho)=O \rho O^{\dagger}$ with $O$ being singleor multiqubit operations. With spatially localized dephasing noise, the quantum map becomes

$$
\begin{aligned}
\mathcal{E}(p)= & \mathcal{U}_{R_{z}^{\dagger}} \circ \mathcal{E}_{\mathrm{deph}}^{\otimes 3} \circ \mathcal{U}_{\mathrm{MS}^{\dagger}} \circ \mathcal{U}_{R_{y}^{\dagger}(\phi)} \\
& \circ \mathcal{E}_{\mathrm{deph}}^{\otimes 3} \circ \mathcal{U}_{\mathrm{MS}} \circ \mathcal{U}_{R_{z}},
\end{aligned}
$$

where the single-qubit dephasing channel is characterized by $\mathcal{E}_{\text {deph }}(\rho)=(1-p) \rho+p \hat{\sigma}_{z} \rho \hat{\sigma}_{z}$, with $p$ being the single-qubit dephasing rate.

To show the effect of the dephasing channel, we calculate the average fidelity $\overline{\mathcal{F}}\left(\mathcal{E}_{\text {id }}, \mathcal{E}(p)\right)$ between the ideal and the noisy process. As shown in Fig. 7(a), the average fidelity decreases as the single-qubit dephasing rate increases such that it is over $98 \%$ when the dephasing rate $p=0.01$.

We also consider the effect of a depolarizing error acting on both the system and the ancillary qubits. For simplicity, we consider ground state preparation in a two-level system. The depolarizing error acts with probability $\delta$ per unit of time. In this case, $\gamma$ and $\tau$ are carefully chosen to make $\phi_{0}=0$ and $\phi_{1} \leqslant \frac{\pi}{2}$. We consider the completely mixed state $\hat{\rho}_{\text {in }}=$ $\frac{1}{2}(|0\rangle\langle 0|+| 1\rangle\langle 1|)$ as the initial state, although the protocol should work for any initial state with a nonzero overlap with the desired state. After $k$ steps, the unnormalized output state
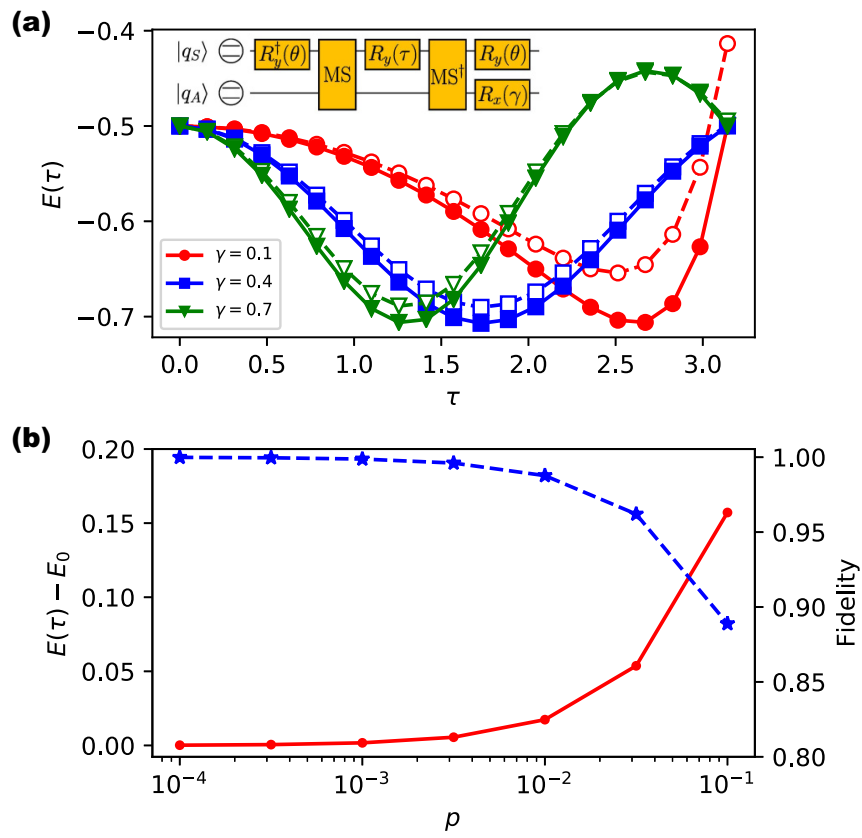

FIG. 8. Cooling of a two-level system under dephasing. The system Hamiltonian is the one in Eq. (C5) with $b_{x}=b_{z}=1$. (a) Average energy after a one cooling step versus the effective evolution time $\tau$. The initial state is $\left|0_{S} 0_{A}\right\rangle$ and the average is taken over the postselected final state. The applied cooling unitary $W_{\gamma}(\tau)$ is depicted in the inset. Solid markers are results obtained in the ideal situation while hollow ones are obtained with dephasing noise. The error rate per MS gate is $p=0.01$. (b) Average energy (solid red) and ground-state fidelity (dashed blue) versus the dephasing strength $p$.

becomes

$$
\mathcal{E}_{\mathrm{de}}^{k}\left(\hat{\rho}_{\mathrm{in}}\right)=\tilde{P}_{0, k}|0\rangle\left\langle 0\left|+\tilde{P}_{1, k}\right| 1\right\rangle\langle 1|,
$$

where the unnormalized probabilities are

$$
\begin{aligned}
& \tilde{P}_{0, k}=(1-\delta) P_{0, k-1}+\frac{\delta}{4}, \\
& \tilde{P}_{1, k}=(1-\delta) P_{1, k-1} \cos ^{2} \phi_{1}+\frac{\delta}{4},
\end{aligned}
$$

with initial conditions $P_{0,0}=P_{1,0}=\frac{1}{2}$. The probabilities for the ground and the excited states can then be derived as $P_{i, k}=$ $\tilde{P}_{i, k} /\left(\tilde{P}_{0, k}+\tilde{P}_{1, k}\right)$ with $i=0$ or 1 .

Figure 7(b) shows that the effective cooling operation changes the stationary state of the system. Instead of retrieving the completely mixed state, which is the stationary state without effective cooling, we end up with a new stationary state, where the excited-state population has been suppressed due to the effective cooling protocol.

Finally, we investigate the effect of dephasing on the cooling of a two-level system. The system Hamiltonian of a spin-1/2 system under a static magnetic field is

$$
\hat{H}_{s}=-\frac{b_{z}}{2} \hat{\sigma}_{z}-\frac{b_{x}}{2} \hat{\sigma}_{x},
$$

where $b_{x}$ and $b_{z}$ are magnetic field components along the $x$ and $z$ axes. We first initialize the system and ancilla qubits to the state $\left|0_{S} 0_{A}\right\rangle$. Then, we implement $\hat{W}_{\gamma}(\tau)$ for some fixed $\tau$ and $\gamma$. Next, we measure the ancilla qubit. The 
quantum circuit implementing $\hat{W}_{\gamma}(\tau)$ is depicted in the inset of Fig. 8(a), where the rotation angle $\theta$ is determined by $\tan \theta=b_{x} / b_{z}$. With dephasing noise, the process is described by the following quantum map:

$$
\begin{aligned}
\mathcal{E}(p)= & \mathcal{U}_{R_{x}(\gamma)} \circ \mathcal{U}_{R_{y}(\theta)} \circ \mathcal{E}_{\mathrm{deph}}^{\otimes 2} \circ \mathcal{U}_{\mathrm{MS}^{\dagger}} \circ \mathcal{U}_{R_{y}^{\dagger}(\tau)} \\
& \circ \mathcal{E}_{\mathrm{deph}}^{\otimes 2} \circ \mathcal{U}_{\mathrm{MS}} \circ \mathcal{U}_{R_{y}^{\dagger}(\theta)} .
\end{aligned}
$$

In Fig. 8, we show the average energy after a cooling step versus the effective evolution time $\tau$, for different shifts $\gamma$. Despite the presence of dephasing, our algorithm still works and lowers the average energy of the system. The obtainable lowest energy, however, is higher than the one achieved in the ideal situation. In Fig. 8(b), we show the residual energy (solid red) and ground-state fidelity (dashed blue) for different noise strengths.
[1] R. P. Feynman, Simulating physics with computers, Int. J. Theor. Phys. 21, 467 (1982).

[2] I. M. Georgescu, S. Ashhab, and F. Nori, Quantum simulation, Rev. Mod. Phys. 86, 153 (2014).

[3] A. W. Harrow, A. Hassidim, and S. Lloyd, Quantum Algorithm for Linear Systems of Equations, Phys. Rev. Lett. 103, 150502 (2009).

[4] D. W. Berry, A. M. Childs, A. Ostrander, and G. Wang, Quantum algorithm for linear differential equations with exponentially improved dependence on precision, Commun. Math. Phys. 356, 1057 (2017).

[5] T. Xin, S. Wei, J. Cui, J. Xiao, I. Arrazola, L. Lamata, X. Kong, D. Lu, E. Solano, and G. Long, Quantum algorithm for solving linear differential equations: Theory and experiment, Phys. Rev. A 101, 032307 (2020).

[6] J. M. Arrazola, T. Kalajdzievski, C. Weedbrook, and S. Lloyd, Quantum algorithm for nonhomogeneous linear partial differential equations, Phys. Rev. A 100, 032306 (2019).

[7] D. S. Abrams and S. Lloyd, Quantum Algorithm Providing Exponential Speed Increase for Finding Eigenvalues and Eigenvectors, Phys. Rev. Lett. 83, 5162 (1999).

[8] M. A. Nielsen and I. L. Chuang, Quantum Computation and Quantum Information (Cambridge University Press, Cambridge, UK, 2000).

[9] E. Farhi, J. Goldstone, S. Gutmann, J. Lapan, A. Lundgren, and D. Preda, A quantum adiabatic evolution algorithm applied to random instances of an NP-complete problem, Science 292, 472 (2001).

[10] A. Aspuru-Guzik, A. D. Dutoi, P. J. Love, and M. Head-Gordon, Simulated quantum computation of molecular energies, Science 309, 1704 (2005).

[11] A. Peruzzo et al., A variational eigenvalue solver on a photonic quantum processor, Nat. Commun. 5, 4213 (2014).

[12] M.-H. Yung et al., From transistor to trapped-ion computers for quantum chemistry, Sci. Rep. 4, 3589 (2014).

[13] J. R. McClean, J. Romero, R. Babbush, and A. Aspuru-Guzik, The theory of variational hybrid quantum-classical algorithms, New J. Phys. 18, 023023 (2016).

[14] A. Kandala et al., Hardware-efficient variational quantum eigensolver for small molecules and quantum magnets, Nature (London) 549, 242 (2017).

[15] Y. Shen, X. Zhang, S. Zhang, J.-N. Zhang, M.-H. Yung, and K. Kim, Quantum implementation of the unitary coupled cluster for simulating molecular electronic structure, Phys. Rev. A 95, 020501(R) (2017).

[16] C. Hempel et al., Quantum Chemistry Calculations on a Trapped-Ion Quantum Simulator, Phys. Rev. X 8, 031022 (2018).
[17] P. J. J. O’Malley, R. Babbush, I. D. Kivlichan, J. Romero, J. R. McClean, R. Barends, J. Kelly, P. Roushan, A. Tranter, N. Ding et al., Scalable Quantum Simulation of Molecular Energies, Phys. Rev. X 6, 031007 (2016).

[18] S. Debnath et al., Demonstration of a small programmable quantum computer with atomic qubits, Nature (London) 536, 63 (2016).

[19] P. O. Boykin, T. Mor, V. Roychowdhury, F. Vatan, and R. Vrijen, Algorithmic cooling and scalable NMR quantum computers, Proc. Natl. Acad. Sci. USA 99, 3388 (2002).

[20] J. Baugh, O. Moussa, C. A. Ryan, A. Nayak, and R. Laflamme, Experimental implementation of heat-bath algorithmic cooling using solid-state nuclear magnetic resonance, Nature (London) 438, 470 (2005).

[21] B. Kraus, H. P. Büchler, S. Diehl, A. Kantian, A. Micheli, and P. Zoller, Preparation of entangled states by quantum Markov processes, Phys. Rev. A 78, 042307 (2008).

[22] J. T. Barreiro et al., An open-system quantum simulator with trapped ions, Nature (London) 470, 486 (2011).

[23] Y. Lin et al., Dissipative production of a maximally entangled steady state of two quantum bits, Nature (London) 504, 415 (2013).

[24] S. Shankar, M. Hatridge, Z. Leghtas et al., Autonomously stabilized entanglement between two superconducting quantum bits, Nature (London) 504, 419 (2013).

[25] F. Verstraete, M. M. Wolf, and J. I. Cirac, Quantum computation and quantum state engineering driven by dissipation, Nat. Phys. 5, 633 (2009).

[26] J.-S. Xu et al., Demon-like algorithmic quantum cooling and its realization with quantum optics, Nat. Photonics 8, 113 (2014).

[27] Y. Li, L.-A. Wu, Y.-D. Wang, and L.-P. Yang, Nondeterministic ultrafast ground-state cooling of a mechanical resonator, Phys. Rev. B 84, 094502 (2011).

[28] P. V. Pyshkin, D.-W. Luo, J. Q. You, and L.-A. Wu, Groundstate cooling of quantum systems via a one-shot measurement, Phys. Rev. A 93, 032120 (2016).

[29] P. V. Pyshkin, D.-W. Luo, J. Q. You, and L.-A. Wu, Nondeterministic quantum computation via ground state cooling and ultrafast Grover algorithm, arXiv:1704.01467.

[30] S. Lloyd, Universal quantum simulators, Science 273, 1073 (1996).

[31] B. P. Lanyon et al., Universal digital quantum simulation with trapped ions, Science 334, 57 (2011).

[32] J. Zhang et al., Observation of a many-body dynamical phase transition with a 53-qubit quantum simulator, Nature (London) 551, 601 (2017). 
[33] A. Safavi-Naini et al., Verification of a Many-Ion Simulator of the Dicke Model Through Slow Quenches across a Phase Transition, Phys. Rev. Lett. 121, 040503 (2018).

[34] K. Toyoda, R. Hiji, A. Noguchi, and S. Urabe, Hong-OuMandel interference of two phonons in trapped ions, Nature (London) 527, 74 (2015).

[35] C. Shen, Z. Zhang, and L.-M. Duan, Scalable Implementation of Boson Sampling with Trapped Ions, Phys. Rev. Lett. 112, 050504 (2014).

[36] J. Casanova, L. Lamata, I. L. Egusquiza, R. Gerritsma, C. F. Roos, J. J. García-Ripoll, and E. Solano, Quantum Simulation of Quantum Field Theories in Trapped Ions, Phys. Rev. Lett. 107, 260501 (2011).

[37] P. H. Leung, K. A. Landsman, C. Figgatt, N. M. Linke, C. Monroe, and K. R. Brown, Robust 2-Qubit Gates in a Linear Ion Crystal Using a Frequency-Modulated Driving Force, Phys. Rev. Lett. 120, 020501 (2018).

[38] E. Solano, Selective interactions in trapped ions: State reconstruction and quantum logic, Phys. Rev. A 71, 013813 (2005).

[39] M. Müller, K. Hammerer, Y. L. Zhou, C. F. Roos, and P. Zoller, Simulating open quantum systems: From many-body interactions to stabilizer pumping, New J. Phys. 13, 085007 (2011).

[40] Y. Lu, S. Zhang, K. Zhang, W. Chen, Y. Shen, J. Zhang, J.-N. Zhang, and K. Kim, Global entangling gates on arbitrary ion qubits, Nature (London) 572, 363 (2019).

[41] J. Casanova, A. Mezzacapo, L. Lamata, and E. Solano, Quantum Simulation of Interacting Fermion Lattice Models in Trapped Ions, Phys. Rev. Lett. 108, 190502 (2012).

[42] E. Solano, R. L. de Matos Filho, and N. Zagury, Entangled coherent states and squeezing in $N$ trapped ions, J. Opt. B: Quantum Semiclassical Opt. 4, S324 (2002).

[43] I. Arrazola, J. S. Pedernales, L. Lamata, and E. Solano, Digitalanalog quantum simulation of spin models in trapped ions, Sci. Rep. 6, 30534 (2016).

[44] J. S. Pedernales et al., Quantum Rabi model with trapped ions, Sci. Rep. 5, 15472 (2015).

[45] R. Puebla, J. Casanova, and M. B. Plenio, A robust scheme for the implementation of the quantum Rabi model in trapped ions, New J. Phys. 18, 113039 (2016).
[46] R. Puebla, M.-J. Hwang, J. Casanova, and M. B. Plenio, Probing the Dynamics of a Superradiant Quantum Phase Transition with a Single Trapped Ion, Phys. Rev. Lett. 118, 073001 (2017).

[47] L. Cong, S. Felicetti, J. Casanova, L. Lamata, E. Solano, and I. Arrazola, Selective interactions in the quantum Rabi model, Phys. Rev. A 101, 032350 (2020).

[48] I. Aedo and L. Lamata, Analog quantum simulation of generalized Dicke models in trapped ions, Phys. Rev. A 97, 042317 (2018).

[49] A. Mezzacapo, J. Casanova, L. Lamata, and E. Solano, Digital Quantum Simulation of the Holstein Model in Trapped Ions, Phys. Rev. Lett. 109, 200501 (2012).

[50] J. S. Pedernales, R. Di Candia, I. L. Egusquiza, J. Casanova, and E. Solano, Efficient Quantum Algorithm for Computing n-time Correlation Functions, Phys. Rev. Lett. 113, 020505 (2014).

[51] R. Di Candia, J. S. Pedernales, A. del Campo, E. Solano, and J. Casanova, Quantum simulation of dissipative processes without reservoir engineering, Sci. Rep. 5, 9981 (2015).

[52] J. J. Sakurai and J. Napolitano, Modern Quantum Mechanics (Addison-Wesley, 2011).

[53] I. I. Rabi, On the process of space quantization, Phys. Rev. 49, 324 (1936).

[54] J. Braumüller et al., Analog quantum simulation of the Rabi model in the ultra-strong coupling regime, Nat. Commun. 8, 779 (2017).

[55] N. K. Langford et al., Experimentally simulating the dynamics of quantum light and matter at deep-strong coupling, Nat. Commun. 8, 1715 (2017).

[56] D. Lv, S. An, Z. Liu, J.-N. Zhang, J. S. Pedernales, L. Lamata, E. Solano, and K. Kim, Quantum Simulation of the Quantum Rabi Model in a Trapped Ion, Phys. Rev. X 8, 021027 (2018).

[57] J. Casanova, G. Romero, I. Lizuain, J. J. García-Ripoll, and E. Solano, Deep Strong Coupling Regime of the Jaynes-Cummings Model, Phys. Rev. Lett. 105, 263603 (2010).

[58] J. Hubbard, Electron correlations in narrow energy bands, Proc. R. Soc. London 276, 238 (1963).

[59] P. Jordan and E. Wigner, Über das paulische Äquivalenzverbot, Eur. Phys. J. A 47, 631 (1928). 